\title{
Complex Network Minority Game Model for the Financial Market Modeling and Simulation
}

\author{
Lingyun Chen (iD) \\ School of Business, Xi'an International University, Xi'an, Shaanxi 710077, China \\ Correspondence should be addressed to Lingyun Chen; chenlingyun@xaiu.edu.cn
}

Received 19 September 2020; Revised 12 October 2020; Accepted 22 October 2020; Published 17 November 2020

Academic Editor: Wei Wang

Copyright (C) 2020 Lingyun Chen. This is an open access article distributed under the Creative Commons Attribution License, which permits unrestricted use, distribution, and reproduction in any medium, provided the original work is properly cited.

\begin{abstract}
This paper proposes a new financial market model based on the analysis of the minority game model. The agent in this model forms a network through information sharing, and the agent uses the minority game model to realize the evolution of the system. To better describe the financial market, we also adopt a prior connection strategy for the model. The network formed by the agent has the characteristics of a scale-free network, and as the initial network connection probability increases, the growth rate of the corresponding agent's average connection degree increases and then decreases after reaching the peak.
\end{abstract}

\section{Introduction}

Among the many complex research fields, the social economy field makes the research more complicated due to the participation of people. The financial market is particularly important in complexity research due to its special nature and unique position in the socioeconomic system [1]. It is precise because of this that it has attracted a large number of scholars from various disciplines. In particular, the entry of physicists, computer scholars, and psychology and behavior scholars has made a research on financial markets gradually intensified. Based on various complexity theories, computational finance came into being a word [2]. It portrays the financial market from the perspective of complex networks, systems, and microcosms. It has become a powerful tool for people to understand and understand the complexity of financial markets, and it has become a new field of financial complexity research [3]. It proposed that the financial market system should be a complex adaptive system (CAS).

Neoclassical economics tells us that the economic system of social existence is composed of many individuals called "economic man". These "economic man" are completely rational and have complete control of all the information on the entire market [4]. The economists proved that the market will eventually be in a state of equilibrium, in which supply and demand are just in equilibrium, and if there is no interference from external factors, the system will always be in equilibrium [5]. However, the actual economic system is different from what economists envisioned in many respects. First of all, the "economic man" individuals in the economy are not omnipotent and fully rational [6]. These individuals are not completely random interactions. These individuals have a learning function. They do not use existing methods to make decisions in the market but gradually accumulate experience through the practice of economic activities and through other economic activities in the limited environment around them. In the interaction of individuals, they gradually adapt to perform bounded rational behaviors $[7,8]$.

In recent years, many empirical studies have also found that financial markets exhibit complex characteristics and market abnormalities. These phenomena can no longer be explained by classical rational expectations theory, efficient market theory, traditional mathematical tools (linear, fixed point, and differential equation system), and traditional equilibrium economic theory. Traditional economic theories have been powerless in the face of reality [9]. To better simulate the process of individual and market operations to discover the micro- and macro-potential laws, computational finance scholars have done a lot of research and positive improvements in modeling and simulation. This is 
the result of mutual complement and mutual penetration of various disciplines [10].

With the further expansion of the process of international economic integration, the economic development of all countries in the world has achieved unprecedented development. The financial markets of various countries have also been greatly improved and perfected [11]. Financial services have had a huge impact on people's lives and become an indispensable service in people's lives. With the development of the financial market, new financial products are emerging in endlessly. The continuous emergence of new products has added a lot of uncertainties in the financial markets where the risk factors are already very large [12].

It is precise because of the complexity and variability of the financial market that the financial market is also becoming an important application field of research results in various disciplines [13]. Economists use their unique economic viewpoints to make a lot of fruitful analysis of financial markets and from many basic theories of economics [14]. Physicists and mathematicians use their unique quantitative research methods to quantitatively analyze and model financial markets. The behavioral scientists on the behavior of individuals in financial markets have done more in-depth and detailed analysis, for the financial markets provide a more precise simulation modeling of the behavior of field and computer simulation scholars on the financial markets [15]. Many contributions have also been made, and many scholars in other fields have also done a lot of research on financial markets. The main purpose of these studies is to be able to find an explanation of the objective laws of the financial market, analyze the dynamic evolution process of the financial market, and analyze the common financial phenomena in the financial market to make more in-depth and objective analysis, so that people can obtain more existing utility in the financial market and ultimately form a healthy development of the financial market.

The financial market is a part of human society. Recent studies in human society have shown that there is a complex network phenomenon in the financial market [16]. The percolation model is one of the methods to study complex networks. As shown in Figure 1, the percolation model is a theory due to changes caused by the effects of interconnected research disordered systems. The upper part is an idealized two-dimensional honeycomb-shaped channel network, showing how the liquid tortuously passes through the hexagonal "coffee grounds". The lower part shows the corresponding network diagram [17]. The thick line represents the linkage and several groups are marked, one of which has been marked as a possible percolation pathway.

The complex network characteristics of financial markets make relevant research on percolation models apply [18]. For applying the percolation model to the financial market, each block corresponds to an agent in the financial market, and whether they are connected to determine whether they form a group. Among them, the Cont-Bouchaud model better applies the percolation model to the financial market, uses this to study the herd effect, and concludes that the herd effect is the main reason for the stock market's fat tail and the stock market's bubble. In the CB model, each set represents an agent [19]. During each iteration, the connected edge between any two grid points becomes a connected edge with a certain probability and the connected grid points from a cluster. Each cluster is regarded as an investment group that agrees and adopts the same investment behavior.

Based on the research results of the artificial financial market of a multiagent platform and complex network, combined with the current popular and complex network, we propose a financial market model that MG evolves on a complex network. This article will comprehensively consider micro- and macrofactors, appropriately adjust their influence on the financial market, and reasonably reflect how they affect the market together.

\section{Financial Market Model Construction Based on a Minority Game Model in a Complex Network}

2.1. Market Asset Model Modeling. This system uses market assets to model real-life financial market transaction objects and market activity rules. Market assets refer to the subject matter that is circulated and traded in the market and are the bearers of price fluctuations in the market. Common assets in financial market simulation include stocks and currency. In the actual financial market, an exchange will trade multiple assets at the same time, and the diversity of assets is one of the reasons for the diversity of the financial market. It is necessary to propose a unified model description for various forms of assets.

The circulation of assets is accompanied by the creation and progress of trading rules. A rule is a description of how an asset changes in trading activities, and it is a behavior of an asset. Assets without rules are not assets in the trading market. Rules and assets come together, which is why the two are regarded as a whole and modeled as marketable assets. The rules need to be implemented by market participants. In the financial market, market participants, market participants, and market participation rules are closely related. In the financial simulation model, the same market participant may own multiple assets, and the same asset may be owned by multiple market participants [20]. For example, a certain exchange may trade multiple stocks at the same time, and an investor may hold multiple stocks at the same time. A certain stock may be listed and traded on multiple exchanges at the same time, and a certain stock may be held by multiple investors. The same rule may be applied to multiple assets, and the same asset may also have multiple market rules. For example, a certain exchange adopts a matchmaking mechanism for all stocks, and the certain stock is traded through matchmaking on some exchanges and market-making on some exchanges. A market participant may adopt different rules for different assets, and the same rule may be used by many different market participants.

The strategy library plays a decisive role in the agent. It determines how the agent trades in the financial market, the result of the transaction will surely bring about changes in the agent's attribute status, and the change of parameters will 


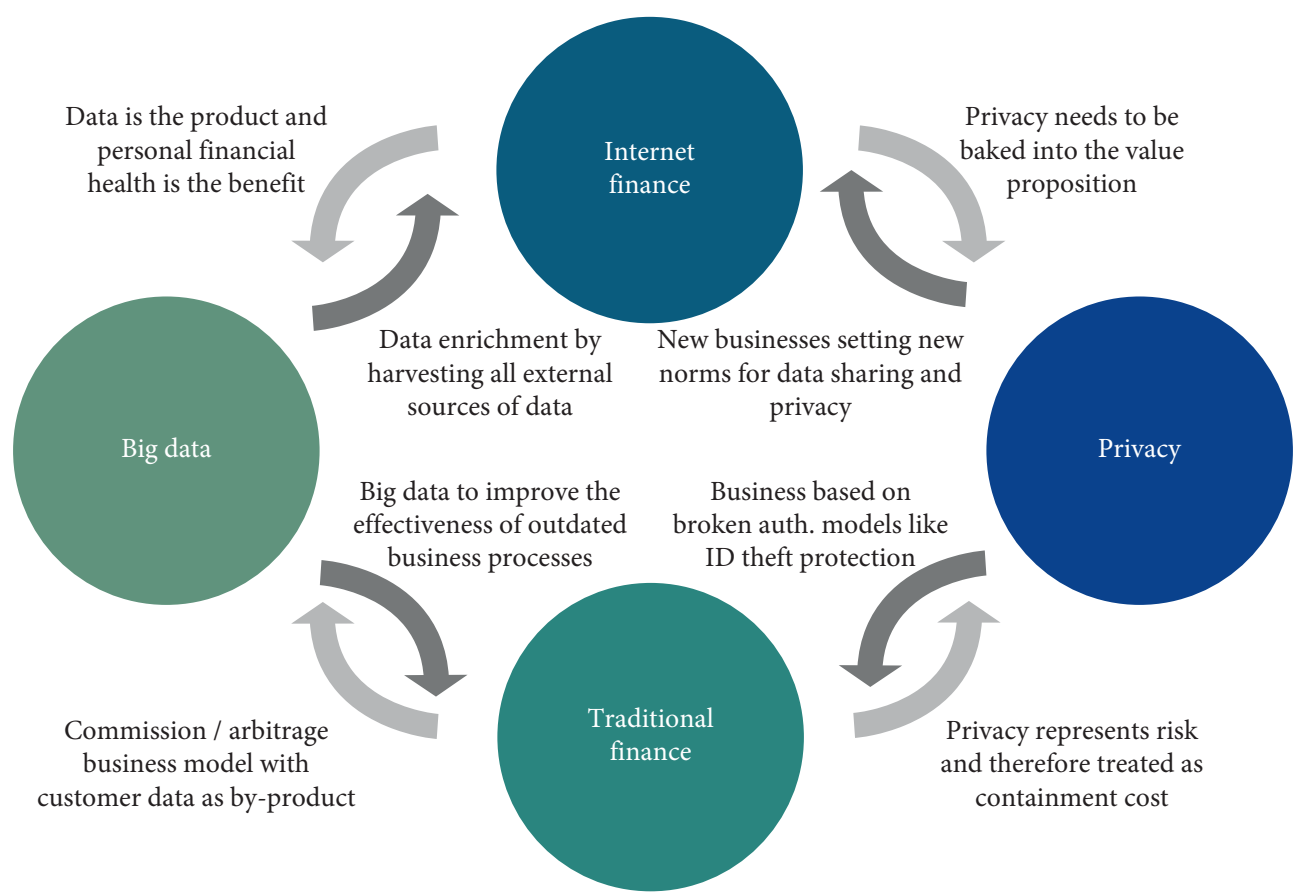

FIGURE 1: Common modeling methods and processes in financial markets.

cause great changes to the agent's links with other agents. The utility function of the agent adopts the utility function of absolute risk aversion:

$$
U\left(w_{i, t}\right)=-\log \left(\lambda \cdot w_{i, t}\right) .
$$

Among them, $\lambda$ is the absolute risk aversion coefficient and $w_{i, t}$ is the wealth of the $i$ th agent at time $t$, including the value of cash and stocks held at that time. Agent's wealth can be expressed by the following formula (2):

$$
w_{i, t}=\sqrt{\sum\left(c_{i, t}+p_{t} h_{i, t}\right)},
$$

where $c_{i, t}$ and $h_{i, t}$ represent the cash and the number of stocks held by the $i$ th agent at time $t$ and $p_{t}$ is the stock price at that time step. In this way, at the next time step, before the agent makes a decision, its wealth state can be expressed by the following formula:

$$
w_{i, t+1}=\frac{c_{i, t}(1+r)}{h_{i, t}\left(p_{t+1}+d_{t+1}\right)} .
$$

Among them, $r$ is the risk-free rate of return and $d_{t+1}$ is the dividend of the stock at the time step. The agent's transaction in the financial market is determined by the agent's strategy library or the strategy with the highest score among the strategies shared. In the previous model, the number of transactions for each agent is one as a unit, buying or selling a unit of stock, which cannot reflect the difference of agents in the financial market. The transaction volume in the model in this article is randomly given within a certain range. For example, the transaction volume randomly takes an integer value from 1 to 10 .

$$
\begin{aligned}
& a_{i}(t)=\left\{\begin{array}{l}
w_{i, t} \cdot \operatorname{random}(0,1), \\
c_{i, t} \cdot \operatorname{random}(50,100),
\end{array}\right. \\
& A(t)=\int_{i=1}^{N} \sum_{i} a_{i, t} .
\end{aligned}
$$

In the financial market, the correct return function is expressed by the following formula:

$$
\begin{gathered}
g(t+1)=\left|a_{i}(t)\right||A(t+1)|, \\
\text { Score }_{i, t}(t+1)=\left\{\begin{array}{l}
\operatorname{Score}_{i, t}(t), \\
\operatorname{Score}_{i, t}(t)+1, \\
\operatorname{Score}_{i, t}(t) \cdot s_{j}(h) .
\end{array}\right.
\end{gathered}
$$

Score $_{i, t}(t+1)$ in represents the integral of the $j^{\text {th }}$ strategy in the $i$ th agent strategy library at time $t+1$. If the prediction of the strategy $s_{j}$ is consistent with the sign of $A(t)$, the agent accumulates the points of the strategy's points. Each timestep agent will select the strategy with the highest accumulated points from its strategy library as the basis for its decision-making:

2.2. Market Participation Theme Model Construction. This system uses the main body of market participants to model various participants in the market, such as investors, exchanges, and various institutions, that affect the state of the market. However, investors have great differences in market behavior. Their responses to market assets and market information have similarities. In this model, these participants are nondistinguished unified abstract model for the 
participants, these design antispecific models come with various possible market-derived abilities to participate.

Market participants are the core part of the financial market and the promotion of the financial market as well as the direct manifestation of intelligent agents in the "multiagent simulation". This model is the core of the general financial market model and even the entire simulation system. In the real financial market, the activities of participants are mainly transaction-related, mainly involving three types of participants, traders, exchanges, and other institutions. Among them, traders and exchanges are the most important part of the financial market and the promotion of the financial market.

Traders use certain strategies to make transactions. The specific performance is to send a transaction order to a specific exchange. In the process of making decisions, traders need to make comprehensive judgments based on their assets, the current price of the subject matter in the market, and other sources of information. The process of obtaining market information and other channel information before making a decision and sending orders to the market after the decision are essentially a process of sending and receiving information to communicate. The decisionmaking behavior of traders is motivated by the passage of time and external time information. An example of merging two incentive models is to regularly invest in the purchase of a certain subject matter when the price of the subject matter falls below the target range. The behavior of constantly checking prices is continuously stimulated by the passage of time, and the behavior of buying occurs after receiving the incentive of price information.

The core role of the exchange is to facilitate the transaction of traders. The exchange accepts order information sent by traders and generates orders based on certain principles. There are many and varied ways for exchanges to generate orders, resulting in different types of exchanges. The exchange is also motivated by the passage of time and external time information [21]. For example, an exchange that adopts a market maker system, after receiving a trade order from a trader, is the counterpart of the trader and completes the transaction process with the trader without performing matching transactions between traders. The quotations of this type of exchange come from the thirdparty market. The exchange needs to monitor the thirdparty's quotations at all times, generate its quotations based on the third-party quotations, and report them to traders. This kind of active monitoring of price information and transaction activities occurs due to the arrival of order information. The composition of other institutions is diverse, and their behavior is also different. These institutions include central banks, commercial banks, and insurance companies. However, these institutions are not the core part of market behavior. They also affect the advancement of market transactions. These institutions have different functions, but their core way to influence the system market is to send messages to the market. For example, the central bank can determine the interest rate situation in the current financial market environment. The interest rate situation represents the risk-free rate of return, which will become one of the influencing factors of traders' trading behavior decisions. The formulation and release of information by these institutions may occur over time or may occur due to external events.

The above analysis has analyzed the commonality of various subjects in behavioral incentives. We analyze the commonalities of various subjects in behavior. Even if the current market participants are mainly investors, they will be different due to their different investment strategies. For example, there may be value investors based on the value of the company in the market, technical investors based on price movements, and new investors who make transactions based on news. Similarly, the diversity of the exchange comes from the diversity of the way it generates orders: the diversity of the subject matter of the exchange and the diversity of the subject matter processing methods [22]. However, from a macroperspective, various investor entities and various exchange entities have a commonality in processing news and then acting on assets based on the news. These commonalities also explain the rationality of unified modeling that treats traders, exchanges, and other institutions in the market as equally intelligent agents.

\subsection{Construction of the Main Body Interaction Network} Model. In reality, individuals in financial markets will never exist in isolation but have complex connections with elements in other markets. The complexity of this connection creates an aspect of the complexity of financial markets. In the financial market, there is a direct relationship between market participants and exchanges. Traders request information from the market, send orders, and market broadcasts information to traders. These are all manifestations of this relationship in specific behaviors. There is a relationship between traders and traders. Traders themselves are people in society, and they are naturally connected to other people in society. This kind of connection brings about the exchange of information and further affects the decisionmaking process of traders in market behavior. The exchange is not limited to being in contact with traders. There will be many exchanges in the market at the same time. The exchanges have different systems and different types of transactions, which will lead to cooperation, competition, and other relationships. Moreover, the exchanges exist as institutions and will be subject to such issues as central banks, regulatory agencies, commercial banks, and other institutions. The impact of these complex network relationships on the financial market itself is an important subject to be studied in the financial field.

A general multiagent simulation platform usually needs to support the network relationship between the agents. The behavior and evolution of the subject in a complex adaptive system need to interact with the outside world, and network relationships are an important basis for their interaction. In the general multiagent simulation model, the network model usually has the characteristics of standardization. For example, when performing space-related multiagent simulation, the interactive network that the agent depends on may be generated by its spatial structure [23]. This kind of 
network has obvious characteristics. The semantics of space also has a regular form. On the other hand, the network in the general multiagent simulation model has the characteristics of agent homogeneity, that is, the different agents connected in the network belong to the same type.

To accurately describe the characteristics of the real financial market, the multiagent platform in the field of financial market simulation needs to make reasonable modeling of the network characteristics of the participating elements in the market. Different from the normalization and homogeneity of the interactive network in the abovementioned general multiagent system model, the interactive network in the financial market model has its characteristics. The connection between market participation elements represented by the interactive network in the financial field is an abstract connection, which has nothing to do with specific meanings such as spatial location. This abstract connection is more random. On the other hand, there are various elements of participation in the financial market. For example, exchanges adopt different transaction strategies, which are different types of entities. In experimental models in the field of financial markets, these elements of different natures often appear together and are linked. Therefore, when modeling the interactive network of market participation elements, a multiagent simulation platform oriented to the financial market field must make a special design for the characteristics of the financial market.

On the one hand, the flexibility of the interactive network model of the simulation platform is a more realistic depiction of the indirect relationship of the network in the real financial market, and on the other hand, it also satisfies the design goal of having good reusability for the financial market simulation platform. The reusability of the simulation platform in the financial field comes from the fact that the generalized model of the platform can be specifically developed to achieve various market activity participation elements with different behaviors and meet different experimental needs, and between various market participation elements, make sufficient combinations to form a rich and diverse model that meets various experimental needs. In the process of combining elements, the relationship of the interactive network is also complicated [24].

This complication comes from two aspects: the increase of the connection between the same kind of elements and the increase of the connection between different kinds of elements. From this, we can conclude that in the complex experimental model of financial markets, the interactive network between market elements includes two types: the network between the same kind of elements and the network between different kinds of elements. As shown in Figure 2, the structure of the interactive network of various elements in a financial market model reflects the characteristics of this interactive network. In this schematic diagram, three elements are participating in financial market activities, represented by squares, circles, and triangles. One possible meaning expressed by the network is the relationship between value investors, technology investors, and multiple stock exchanges. There are interconnected network relationships within the square elements, triangle elements, and circular elements. The solid lines are drawn to indicate the network relationships within the same investors or the influence relationships between various exchanges. There are also interconnected network relationships between square elements and triangle elements, sound elements and triangle elements, square elements, and triangular elements, which are drawn with dotted lines to indicate the value investors and technical investors in various exchanges. Account opening relationship communicated value investors and technology investors.

In the simulation of financial markets, possible interactive networks include a ER random network model, a regular network model, a small world network, and a scalefree network. These complex network models are essentially directed graphs, and their directionality comes from the oneway transmission of information in the financial market. As shown in Figure 3, investors can subscribe to news from the media but not vice versa. For this kind of directed graph with multiple node elements, the first method is to use an adjacency list with element types to describe it.

\section{Results and Analysis}

\subsection{Common On-Site Analyses of Financial Markets Based on} the Minority Game Model of Complex Networks. The result of the experiment is given to the simulation of the financial market by the model simulation program in this paper. The number $N$ of agents in the artificial financial market is 1001, the memory capacity of the MG model is 5 , and experiments are performed on each one. The initial stock price in the financial market is 100.0, and the initial wealth and cash of each agent are set. The initial parameters related to network evolution are as follows: the initial network connection probability is 0.05 and the system value and the probability formula for the dynamic evolution process are 0.05 .

When the memory capacity of the MG model is 3 , the simulation program can always run for the stock price time series generated by our model. It shows the stock price time series of the first 4000 iterations. It can be seen that the simulated stock price sequence is very similar to the trend and fluctuation of the real financial market stock price.

When the memory capacity of the MG model is 4 , the time series of stock price returns as generated by our model. As shown in Figure 4, major events (large price changes) in our model occur frequently, which is consistent with the empirical research of many scholars, but neoclassical economic theories cannot explain these phenomena.

Figure 5 shows the probability distribution of the logarithmic return of the stock price generated by our model, when the memory capacity of the MG model is 5 . The fitting curve is a probability curve of normal distribution. As shown in Figure 6, the probability distribution of stock price logarithmic return is very different from the normal distribution. The distribution generated by the model is sharper than the Gaussian distribution at the center, the two ends of the distribution are fatter than the Gaussian distribution, and the probability is not approximately equal to 0 . 


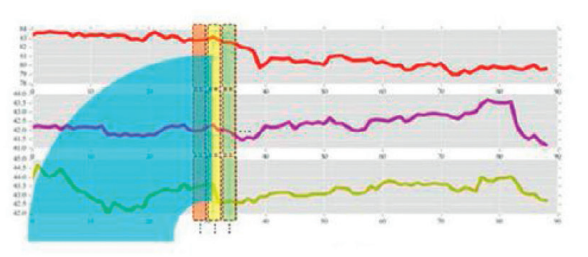

$I\left(X_{i} ; Y_{j}\right)=\sum p(x, y) \log ((p(x, y) /(p(x) p(y))$
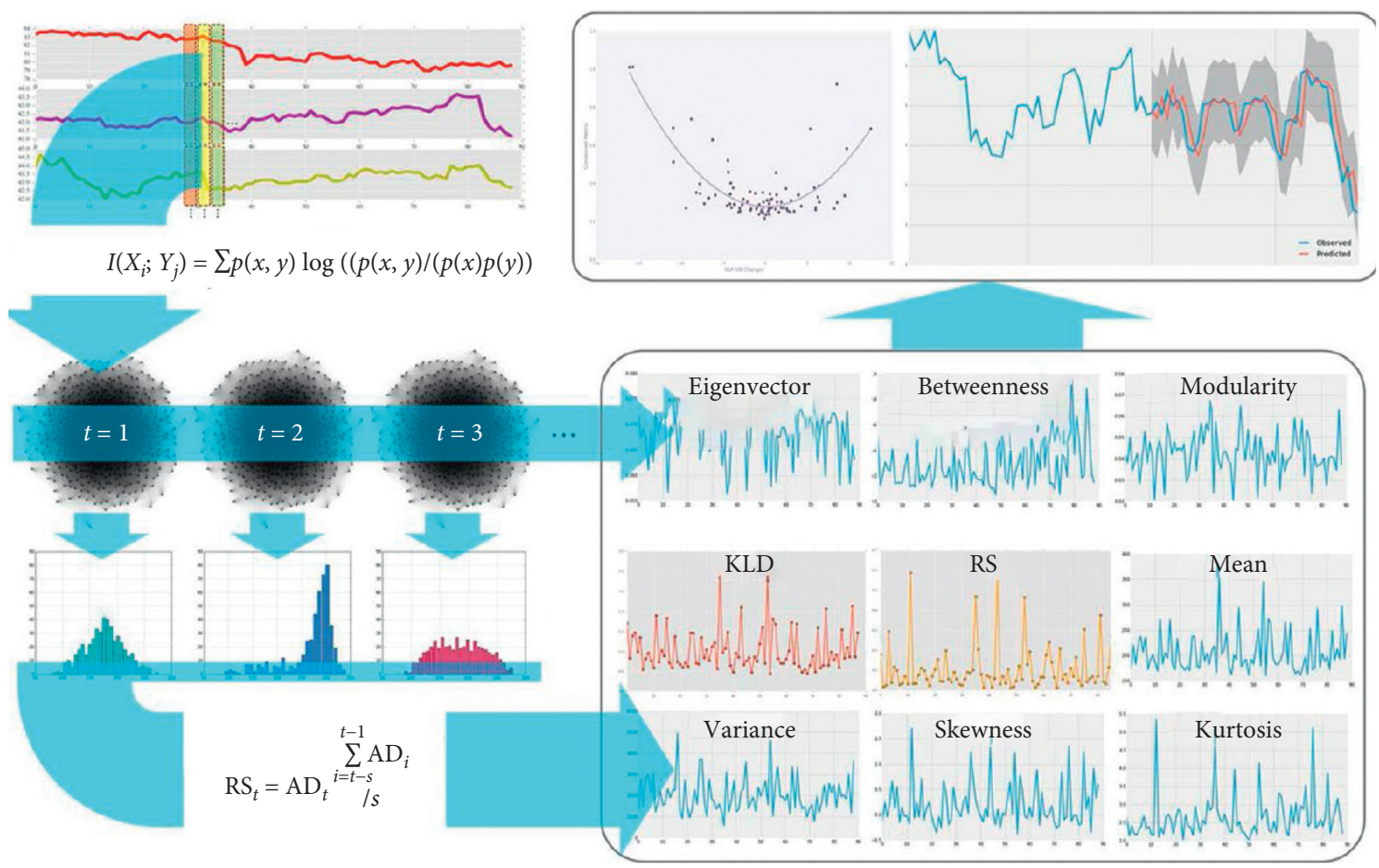

FIGURE 2: Complex network minority game model financial market modeling process.

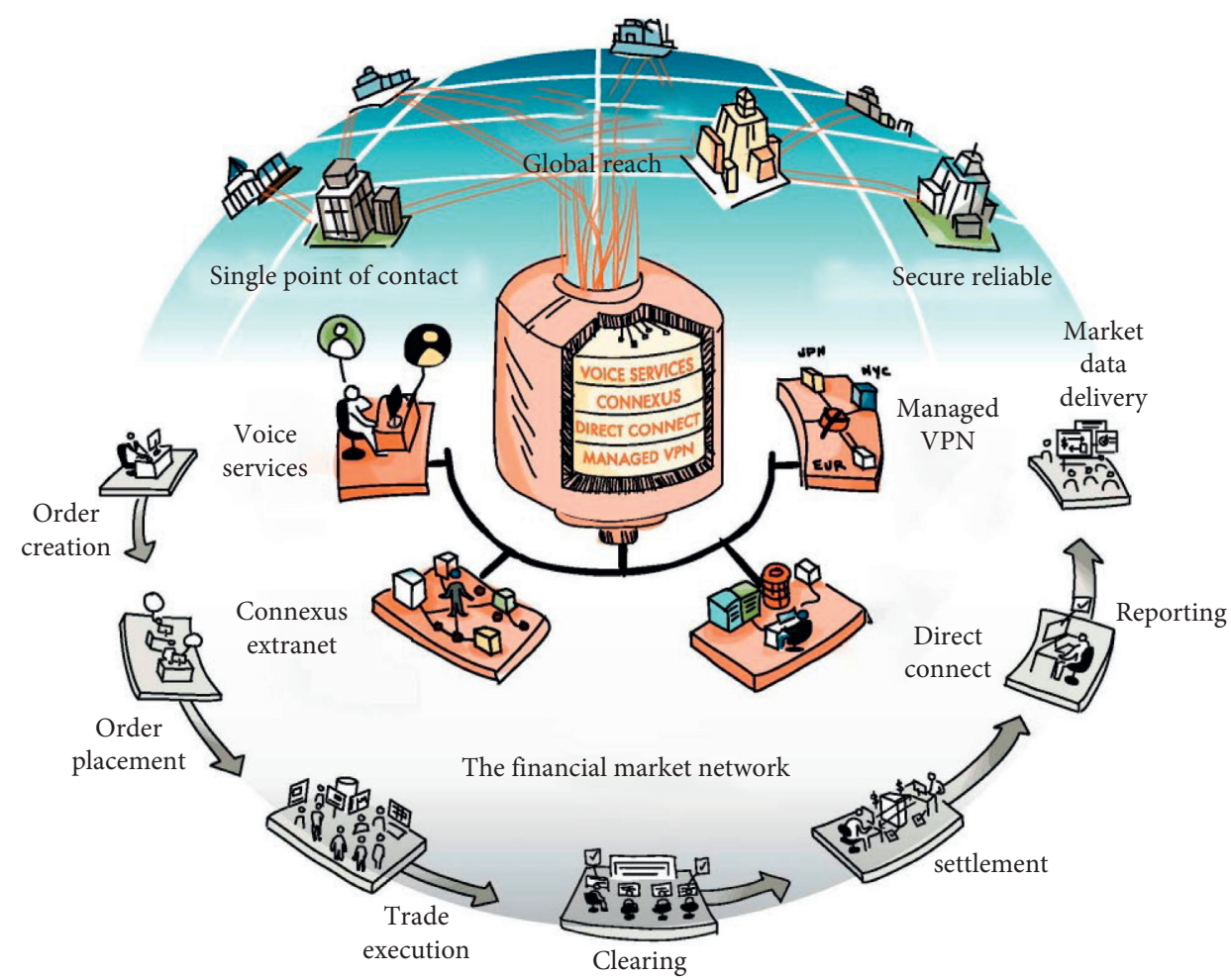

Figure 3: Interactive network of financial market elements.

3.2. Analysis of the Evolution of the Computer Simulation Network Structure of the Financial Market. In this study, experimental data were obtained through a model simulation program, and these data were analyzed, and significant results were obtained. First, it analyzes the common phenomena in the financial market, such as the frequent occurrence of major events in prices and returns and the "spike and thick tail" characteristics of the income distribution. 


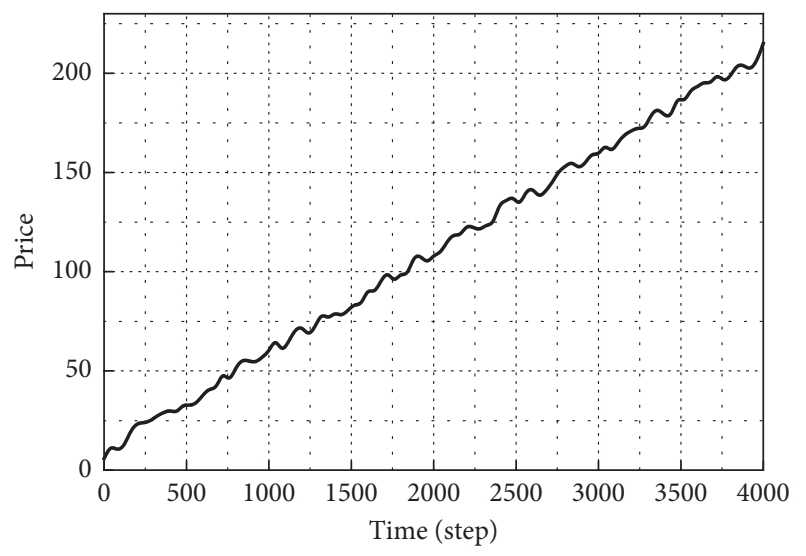

Figure 4: The trend of stock prices in the financial market.

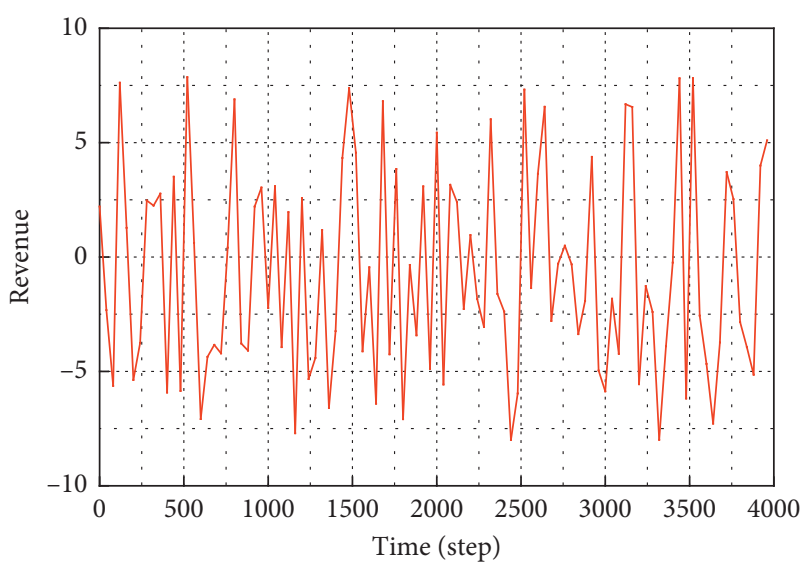

FIgURE 5: Time series of earnings.

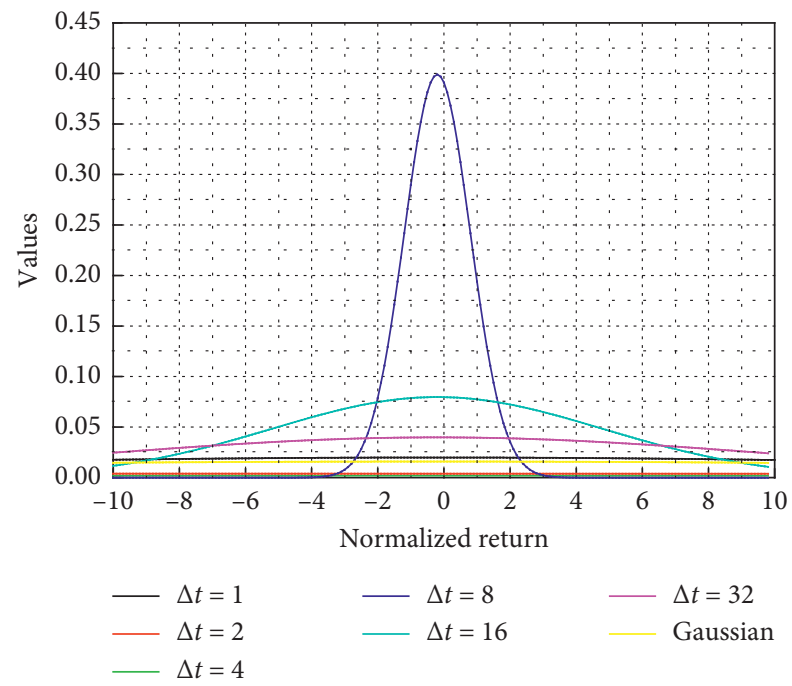

FIGURE 6: Financial market income distribution.

These analytical results are consistent with some common phenomena in empirical research. Then, the dynamic evolution of the complex network structure is analyzed through experimental results. Agent out-degree and in-degree were analyzed and the agent can approximate a straight line logarithmic coordinates, which is consistent with the phenomenon of scale-free complex networks [25]. At the same time, it is also found that there are a certain number of agents with relatively large degrees, which is consistent with the "following effect" and "herding effect" in empirical research. Besides, it finds that when the initial probability increases. The average growth rate of the corresponding agent connection degree increases first and then slows down.

From the simulation program, the network formed by the agents of the process needs the financial market. Through the analysis and processing of these data, the following conclusions are drawn. The probability distribution of outdegree and in-degree chose 3000 hour-step operations. As shown in Figure 7 , the straight line is a straight line on logarithmic coordinates. It can be seen that the probability distribution of out-degree and in-degree in logarithmic coordinates can be approximated by a straight line on logarithmic coordinates, which is consistent with the characteristics of the scale-free network.

The straight line in the probability distribution diagram of the out-degree and in-degree of all agents is a straight line on the logarithmic coordinate. It can be seen that the probability distribution of out-degree and in-degree in logarithmic coordinates can be approximated by a straight line on logarithmic coordinates, which is also consistent with the characteristics of the scale-free network.

The agent network generated by our model contains the characteristics of a scale-free network, so the network is a scale-free network. It can be seen that agents with relatively large degrees still exist, which shows that there are many agents connected to it. This is also consistent with the "following effect" and "herding effect" in empirical research.

The change of the initial network connection probability will affect network evolution. The $x$-coordinate is the increase of the probability from 0.05 to 0.85 with a step length of 0.05 , and their coordinate is the average out-degree and average in-degree of all agents at any time step. It can be seen that, as the initial connection probability increases, the growth rate of the average out-degree and the average indegree increases first and then slows down.

To verify that the distributed simulation platform studied in this article has good performance, we have completed the number of investor agents in the case of 2 calculation nodes, 3 calculation nodes, 4 calculation nodes, and 5 calculation nodes. There are multiple simulation experiments of 200,400,800, and 1600, respectively. The running timeline chart obtained by the simulation experiment. As shown in Figure 8, as the scale of the storage increases, the time required to complete the simulation also shows an upward trend. For the same number of investor Agents, more calculation nodes are used; more simulation consumed shorter running time. This shows that the parallel operation has accelerated the progress of the simulation.

This article discusses the reusability of the simulation system of two aspects, model level and code level, on the reusability of test certificate also from two layers of departure. The so-called system has been successfully reused, meaning that even if the original model and code are used 


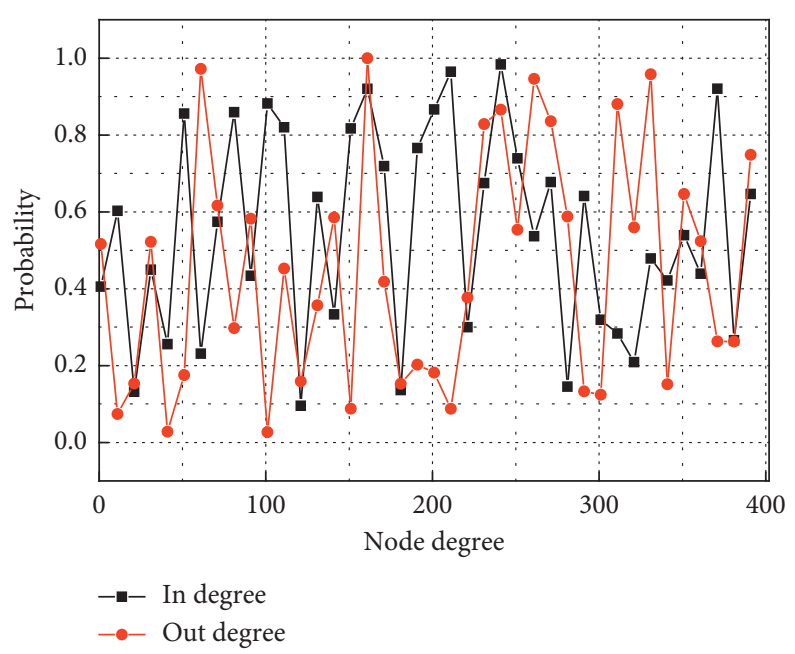

FIgURE 7: Probability distribution of node degree in the financial market.

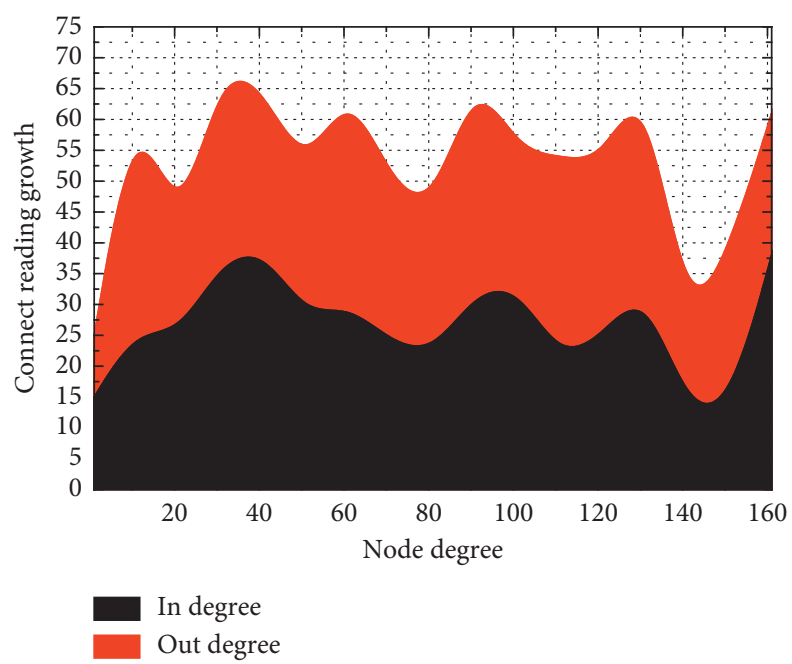

FIGURE 8: The relationship between the initial connection probability and the average increase of the agent out-degree and in-degree.

with a small amount of modification, it is applied to the new model and code, which are in a process of inheriting and deriving the original model code. Therefore, the design of reusability experiments needs to be modified from the original model. If the reconstructed model runs successfully and the expected effect is successful, it can indicate that the reuse activity brought about by the original model is successful, which in turn indicates that the original model or code is reusable and the system is reusable.

To verify the reusability of the simulation system, that is, the simulation system can be modified as little as possible to adapt to the new simulation model, this paper designs the following simulation experiment. This experiment transforms it based on the built-in stochastic market model in the system. The new experimental model adds a new stock asset to the original exchange based on the original model. At the same time, a new exchange is added, which has a stock asset. The two stock exchanges adopted different methods of matching transactions with different maximum single-day limits on the three stocks. Investors randomly place orders at random prices for a certain asset on an exchange. After simulation experiments, the simulated prices of the three stocks in the two exchanges are shown in Figure 9.

It can be seen from Figure 10 that the volatility of different stocks on the two exchanges with a price limit of $10 \%$ is lower than that of the stocks with a price limit of $20 \%$, and the price changes of the three stocks are independent of each other. The three subgraphs show the volatility of the above three stocks. The calculation method is the percentage of the price difference between the current cycle price and the previous cycle price in the previous cycle price.

As shown in Figure 11, the two stocks are on two different exchanges, but both have the same rise and fall limits. The volatility of the two is within the range defined by the matching algorithm, and the absolute value of the volatility is up to $10 \%$. Two stocks are on the same exchange, and each has a different price limit. For serial number 1 stock with a price limit of $10 \%$, the absolute value of the maximum 


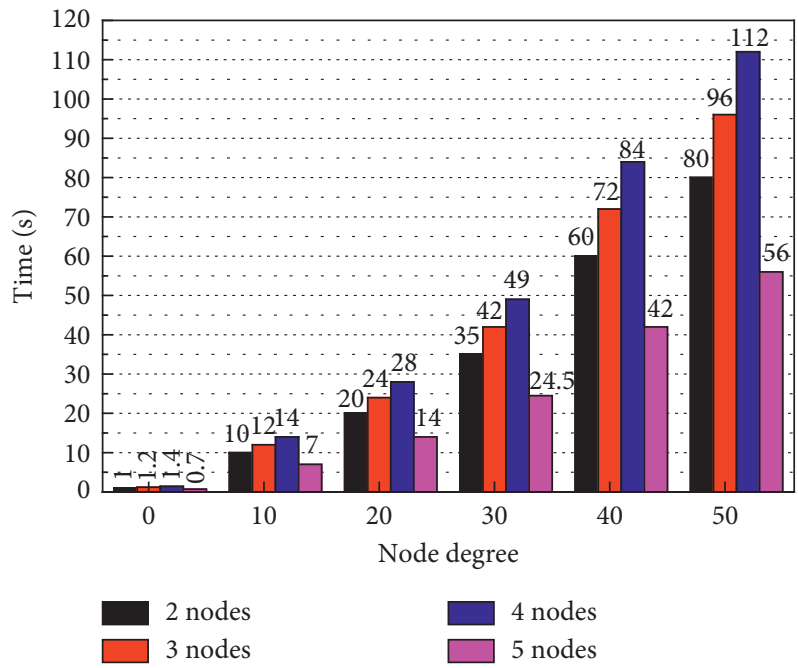

Figure 9: Simulation running time of system effectiveness experiment.

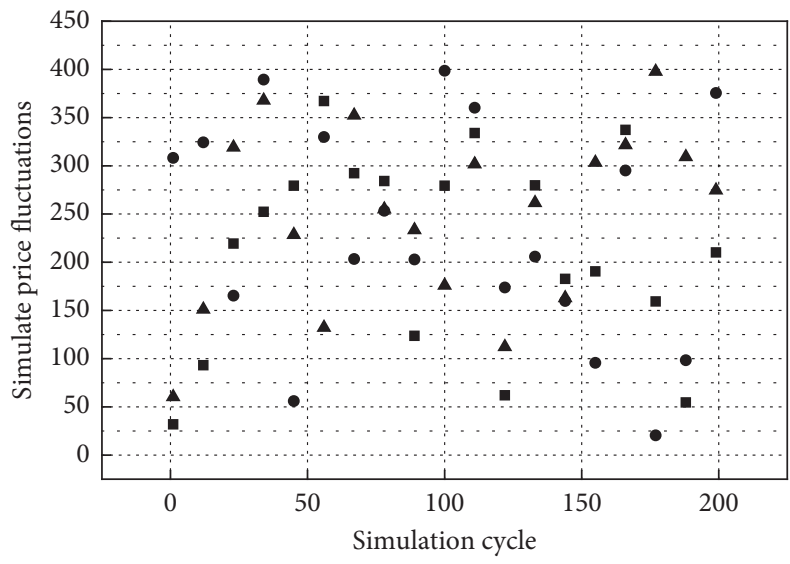

- No. 1

- No. 2

A No. 3

FIGURE 10: System reusability experiment stock price trend.

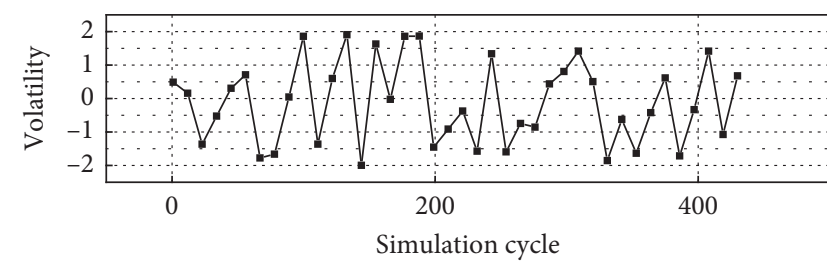

$\rightarrow-$ No. 1

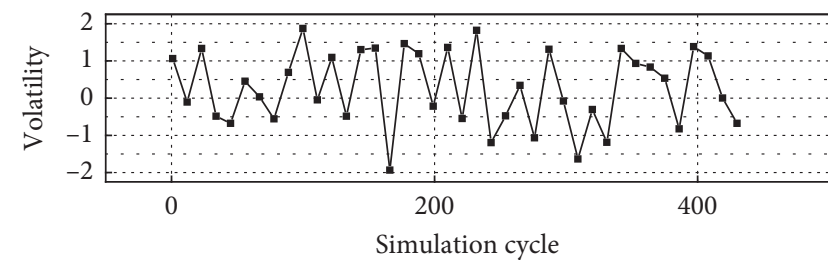

- - No. 2

(b)

(a)

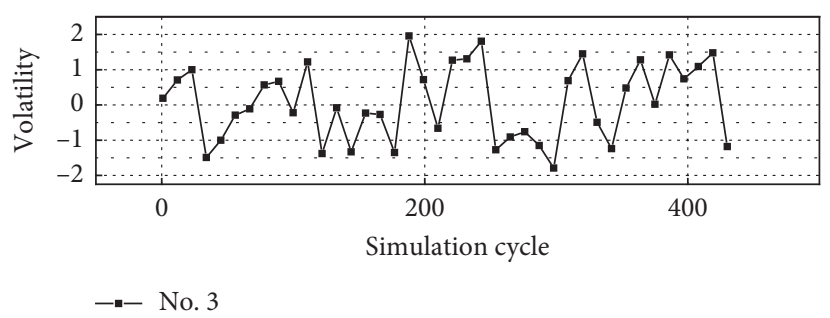

(c)

FIgURE 11: System reusability experiment stock volatility changes. 
volatility is $10 \%$. For serial number 2 stocks with a price limit of $20 \%$, the absolute value of the maximum volatility is $20 \%$. The volatility of the two stocks is within the limits of their respective matching algorithms and is different from each other. This shows that different matching algorithms are effective.

Based on the analysis, the new experimental model is obtained by adding market model elements based on the original experimental model and is a repeated use process of the original experimental model from model to code. In the new experimental model, the price trends of stocks with the same asset processing algorithm between different exchange entities are independent of each other, but they all follow the matching algorithm within their respective limits, indicating that different entities, different assets, and different algorithms operate normally. Compared with the random market model, this experimental model also increases the process of communication between subjects. Two transactions within the investor agent are interconnected. Investor agent constructs and sends a text message to all agents connected to it before making the decision-making process of the order. This message does not affect the recipient's decision on the order.

\section{Conclusion}

The model proposed in this paper is an artificial financial market model based on MG and complex network theory. First, the minority game model more realistically simulates the psychological and behavioral factors of the agents in the financial market and makes financial decisions with the help of the strategy library. Secondly, the complex network formed between the agents is proposed, and the network analyzed agent's information-sharing mechanism and the dynamic evolution process of the network. The simulation research paper has also made some achievements, such as the simulation data obtained through analysis, stock prices and earnings of large events frequently, and "fat tail" feature income distribution; these findings are consistent with those of empirical research. This article analyzes the complex network structure and evolution formed between agents, and it is concluded that the out-degree and in-degree distributions of the agents approximately conform to the power-law distribution, which is consistent with the scalefree phenomenon in complex networks and is also consistent with the "following effect" and the "herding effect". This article analyzes the influence on the network evolution when the initial connection probability of the network changes. As the initial connection probability increases, the corresponding agent average connection degree increases. The speed increases first and then decreases after reaching the peak. The research on the artificial financial market is a huge project, and many factors need to be considered. The model in this article puts forward some meaningful and active explorations on the agent's psychology, behavior, and network formed by the agent, and many areas need to be further improved. The simulation of the psychology and behavior of traders in the financial market by the minority game model should be more perfect. The agent simulated formation of a complex network requires further study and more accurate simulation agent interconnection. Agent complex networks formed a deeper lack of theoretical research and modular approach.

\section{Data Availability}

The data used to support the findings of this study are available from the corresponding author upon request.

\section{Conflicts of Interest}

The authors declare that they have no conflicts of interest regarding the publication of this paper.

\section{Acknowledgments}

This work was supported by the 2019 Xi'an Social Science Planning Fund: Research on the Economic Effects of Xi'an Exhibition Industry (Project Number: 19J01).

\section{References}

[1] S. Ramachandra, S. Maghsudi, and E. Hossain, "Minority game with applications to distributed decision making and control in wireless networks," IEEE Wireless Communications, vol. 24, no. 5, pp. 184-192, 2017.

[2] R. Damaševičius and D. Ašeriškis, "Visual and computational modelling of the minority games," Technology, Education, Management, Informatics, vol. 6, no. 1, pp. 108-116, 2017.

[3] G. I. Brioschi and U. Merlone, "Evolutionary minority game with memory," Journal of Evolutionary Economics, vol. 27, no. 5, pp. 859-875, 2017.

[4] P. Młodzianowski and D. Młodzianowski, "Identification of areas of econophysical models application," Economics, vol. 6, no. 1, pp. 34-44, 2018.

[5] A. S. Chakrabarti and D. Ghosh, "Emergence of anti-coordination through reinforcement learning in generalized minority games," Journal of Economic Interaction and Coordination, vol. 14, no. 2, pp. 225-245, 2019.

[6] H. Huang, Y. Cai, H. Xu et al., "Multivalent minority-gamebased demand-response management of smart buildings toward peak load reduction," IEEE Transactions on ComputerAided Design of Integrated Circuits and Systems, vol. 36, no. 4, pp. 573-585, 2016.

[7] S.-P. Zhang, J.-Q. Zhang, Z.-G. Huang, B.-H. Guo, Z.-X. Wu, and J. Wang, "Collective behavior of artificial intelligence population: transition from optimization to game," Nonlinear Dynamics, vol. 95, no. 2, pp. 1627-1637, 2019.

[8] D. Ašeriškis and R. Damaševičius, "Computational evaluation of effects of motivation reinforcement on player retention," Journal of Universal Computer Science, vol. 23, no. 5, pp. 432-453, 2017.

[9] F. Saffron, G. Gianini, H. Hildmann et al., "Long-term memory-induced synchronisation can impair collective performance in congested systems," Swarm Intelligence, vol. 13, no. 2, pp. 95-114, 2019.

[10] L. Cao, "Research on two decision models in third-party payment platform transaction," Wireless Personal Communications, vol. 110, no. 1, pp. 141-151, 2020.

[11] W. Lu, J. Wang, and C. Xia, "Role of memory effect in the evolution of cooperation based on spatial prisoner's dilemma 
game," Physics Letters A, vol. 382, no. 42-43, pp. 3058-3063, 2018.

[12] A. G. Haldane and A. E. Turrell, "Drawing on different disciplines: macroeconomic agent-based models," Journal of Evolutionary Economics, vol. 29, no. 1, pp. 39-66, 2019.

[13] J. P. Buchanan, "Coelophysis: still fringe after 30 years?" Eurovision News, vol. 50, no. 1, pp. 24-27, 2019.

[14] J. Yang and A. Carro, "Two tales of complex system analysis: MaxEnt and agent-based modeling," The European Physical Journal Special Topics, vol. 229, no. 9, pp. 1623-1643, 2020.

[15] B. Lebanon, "Financial prices dynamics and agent-based models as inspiring by benefit Mandelbrot," The European Physical Journal Special Topics, vol. 225, no. 17, pp. 32433254, 2016.

[16] S. C. Brailsford, T. Eldabi, M. Kunc, N. Mustafee, and A. F. Osorio, "Hybrid simulation modelling in operational research: a state-of-the-art review," European Journal of Operational Research, vol. 278, no. 3, pp. 721-737, 2019.

[17] A. G. Haldane and A. E. Turrell, "An interdisciplinary model for macroeconomics," Oxford Review of Economic Policy, vol. 34, no. 1-2, pp. 219-251, 2018.

[18] M. Melissa, "Application of collective knowledge diffusion in a social network environment," Enterprise Information Systems, vol. 13, no. 7-8, pp. 1120-1142, 2019.

[19] K. Shafi and H. A. Abbass, "A survey of learning classifier systems in games (review article)," IEEE Computational Intelligence Magazine, vol. 12, no. 1, pp. 42-55, 2017.

[20] Y. Wan, B. Ma, and Y. Pan, "Opinion evolution of online consumer reviews in the e-commerce environment," Electronic Commerce Research, vol. 18, no. 2, pp. 291-311, 2018.

[21] M. Zhan, H. Liang, G. Kou, Y. Dong, and S. Yu, "Impact of social network structures on uncertain opinion formation," IEEE Transactions on Computational Social Systems, vol. 6, no. 4, pp. 670-679, 2019.

[22] Z. Chen, "Economic benefit analysis of green building based on fuzzy logic and bilateral game model," Journal of Intelligent \& Fuzzy Systems, vol. 37, no. 1, pp. 301-313, 2019.

[23] Z. He, T. C. E. Cheng, J. Dong, and S. Wang, "Evolutionary location and pricing strategies for service merchants in competitive $\mathrm{O} 2 \mathrm{O}$ markets," European Journal of Operational Research, vol. 254, no. 2, pp. 595-609, 2016.

[24] T. A. Huber and D. Sornette, "Can there be a physics of financial markets? Methodological reflections on econometrics," The European Physical Journal Special Topics, vol. 225, no. 17-18, pp. 3187-3210, 2016.

[25] S. A. Yoon, C. Evans, K. Miller, E. Anderson, and J. Koehler, "Validating a model for assessing science teacher's adaptive expertise with computer-supported complex systems curricula and its relationship to student learning outcomes," Journal of Science Teacher Education, vol. 30, no. 8, pp. 890-905, 2019. 